cytology (ASC-H or HSIL) in 6/9 patients. There was 1 newly diagnosed HIV infection. There were 6 incident syphilis infections. Demographic and sexual behaviour data and correlates of HGAIN will be presented.

Conclusion High risk anal HPV infection and HGAIN was highly prevalent at baseline within this cohort of Asian MSM reinforcing the importance of screening within this population.

Disclosure of interest The study was funded by grant from TreatAsia.

\section{P17.18 FACTORS PREDICTING THE OCCURRENCE OF TUBERCULOSIS FOLLOWING INITIATION OF HIGHLY ACTIVE ANTIRETROVIRAL THERAPY (HAART)}

B Achappa*. Department of Internal Medicine, KMC, Mangalore Affiliated to Manipal University

\subsection{6/sextrans-2015-052270.596}

Background HIV infection is the greatest risk factor for progression of latent Tuberculosis (TB) infection to active TB. Co-infection with these two pathogens is the greatest cause of death in HIV/AIDS patients. TB may develop or latent TB may become active following initiation of HAART. This study was done to evaluate risk factors contributing to development of active TB following initiation of HAART, to detect prevalence of latent TB in treatment naïve patients and to correlate latent $\mathrm{TB}$ at initiation of HAART to development of active TB during first year of treatment.

Methods This hospital based follow up study done over two years period (2013-14) included 48 treatment naïve patients initiated on HAART (2NRTI +1NNRTI). All subjects were screened at baseline for CD4 count, ESR, Quantiferon TB Gold test, BMI, past history of $\mathrm{TB}$ and followed up every three months for 1 year. Data collected was analysed using SPSS Version 11.5 statistical software.

Results $79 \%$ of patients were $31-50$ years of age. 30 were males and 18 females.

$20.83 \%$ patients developed TB within 1 year of initiation of HAART. 23(47.9\%) had latent TB (positive Quantiferon TB Gold test). Of these, 8 (30\%) patients eventually developed TB, whereas $2(8 \%)$ of patients with negative Quantiferon TB Gold test developed TB which was statistically significant.

$7(70 \%)$ patients who developed active $\mathrm{TB}$ at end of study had CD4 T-cell count of less than $200 / \mu \mathrm{l} .13$ out of 23 patients of latent TB and 6 out of 10 with active TB had BMI $<18.5 .7$ out of 18 latent TB patients who developed active TB had ESR above $40 \mathrm{~mm} / 1^{\text {st }} \mathrm{Hr}$.

Conclusion Prevalence of latent TB is high in patients being initiated on HAART.

TB is more likely to develop in patients with lower BMI, higher ESR, lower CD4 count and Quantiferon TB Gold test positivity at baseline after initiation of HAART.

Disclosure of interest None.

\section{P17.19 TIME TO LINKAGE, RETENTION AND ADHERENCE TO HIV CARE AMONG MARRIED COUPLES IN THE FISHING COMMUNITIES ON LAKE VICTORIA}

Zachary Kwena*, Phoeba Olugo, David Angawa, Elizabeth Bukusi. Research, Care and Training Program, Kenya Medical Research Institute, Nairobi, Kenya

$10.1136 /$ sextrans-2015-052270.597
Background Prompt linkage to care, retention in care and adherence to medication are important in achieving the WHO's 9090-90 treatment target. Achieving these targets is important for treatment and prevention of new infections. We sought to establish the time to linkage to care, retention in care and adherence to HIV medications in a cohort of people testing HIV positive in the fishing communities on Lake Victoria.

Methods We conducted a 2-year resurvey of 128 participants who had tested HIV positive in a previous study. These participants were asked to return to the study clinic to participate in a follow-up study. Returning participants were consented and invited for a face-to-face interview on enrolment into HIV care, time to enrolment, retention in care and adherence to HIV care instructions. We mainly used descriptive statistics to analyse the data.

Results Of 128 participants, $47.7 \%$ were female and half were aged 33.5 (IQR, 29-38.7) with a median monthly income of \$66 (IQR, 33-99). Eighty three percent had enrolled in HIV care within a median of 20 days (IQR, 7-60) after testing HIV positive. Women were more likely to enrol in care compared to men $(91.8 \%$ vs $74.6 \% ; \mathrm{p}=0.011)$ but no significant difference in time taken to enrol in care. Over $38 \%$ of the participants had ever missed their clinic appointments since they first enrolled into care with half having missed 2 appointments (IQR, 1-3.5) in the preceding 12 months. Similarly, 26\% had ever missed taking medications as prescribed with half having missed twice (IQR, 1-8.5) in the preceding 12 months. The most common reason cited for both missing clinic appointments and taking medications prescribed was travelling away from home.

Conclusion The numbers enrolled into care falls below the expected 90\% to achieve 90-90-90 treatment target by WHO. More innovative ways need to be developed to achieve these targets.

Disclosure of interest statement This research was supported by a grant from the Consortium for National Health Research. No pharmaceutical grants were received in the development of this study.

\section{P17.20 PATIENT PERSPECTIVES ON THE HIV TREATMENT CASCADE IN THE UNITED KINGDOM}

${ }^{1} \mathrm{~T}$ Rai, ${ }^{1} \mathrm{~J}$ Bruton, ${ }^{2} \mathrm{C}$ Higgs, ${ }^{2} \mathrm{~J}$ Rowlands, ${ }^{1} \mathrm{H}$ Ward*. ${ }^{1}$ Imperial College London; ${ }^{2}$ Chelsea and Westminster NHS Foundation Trust, London

\subsection{6/sextrans-2015-052270.598}

Introduction Figures for the UK's HIV treatment cascade are among the best worldwide with over 95\% retention once in care, however guidelines and service models are changing. We examine perspectives on each stage of the cascade among four generations of patients.

Methods In-depth interviews with $48 \mathrm{HIV}$-positive adults from two clinics. Participants were purposively selected from the four 'HIV generations', based on ART development - those diagnosed pre-1996, 1997-2005, 2006-2012, and since 2013. Framework was used to analyse the data.

Results Diagnosis - Participants from the pre-treatment era were diagnosed on the development of AIDS-defining symptoms, or following a partner's diagnosis. Late diagnoses more recently were because patients underestimated their own risk or failures of healthcare professionals to spot indicator conditions.

Linkage with care - Earlier generations sometimes disengaged with care for a period following diagnosis, dismayed by limited 